\title{
New Method for Estimating Fractal Dimension in 3D Space and Its Application to Complex Surfaces
}

\author{
Matej Babič\#, George Ch. Miliaresis^, Matjaž Mikošs, Rita Ambu ${ }^{\&}$, Michele Calì \\ ${ }^{\#}$ Faculty of Information Studies, Ljubljanska cesta 31a, 8000 Novo mesto, Slovenia \\ E-mail:babicster@gmail.com
}

`Open University of Cyprus, PO Box 12794, 2252 Nicosia, Cyprus

E-mail: miliaresis.g@gmail.com

${ }^{\$}$ Faculty of Civil and Geodetic Engineering, University of Ljubljana, Jamova cesta 2, Ljubljana, Slovenia E-mail:Matjaz.Mikos@fgg.uni-lj.si

\author{
${ }^{\&}$ Department of Mechanical, Chemical and Materials Engineering, University of Cagliari, Via Marengo 2, Cagliari, 09123, Italy \\ E-mail:ambu@unica.it
}

*Electric, Electronics and Computer Engineering Department, University of Catania, viale A. Doria 6, Catania, 95125, Italy E-mail:michele.cali@dieei.unict.it

\begin{abstract}
The concept of "surface modeling" generally describes the process of representing a physical or artificial surface by a geometric model, namely a mathematical expression. Among the existing techniques applied for the characterization of a surface, terrain modeling relates to the representation of the natural surface of the Earth. Cartographic terrain or relief models as threedimensional representations of a part of the Earth's surface convey an immediate and direct impression of a landscape and are much easier to understand than two-dimensional models. This paper addresses a major problem in complex surface modeling and evaluation consisting in the characterization of their topography and comparison among different textures, which can be relevant in different areas of research. A new algorithm is presented that allows calculating the fractal dimension of images of complex surfaces. The method is used to characterize different surfaces and compare their characteristics. The proposed new mathematical method computes the fractal dimension of the 3D space with the average space component of Hurst exponent $\mathrm{H}$, while the estimated fractal dimension is used to evaluate, compare and characterize complex surfaces that are relevant in different areas of research. Various surfaces with both methods were analyzed and the results were compared. The study confirms that with known coordinates of a surface, it is possible to describe its complex structure. The estimated fractal dimension is proved to be an ideal tool for measuring the complexity of the various surfaces considered.
\end{abstract}

Keywords — image analysis; fractal dimension; surface; space component; hurst exponent $\mathrm{H}$.

\section{INTRODUCTION}

Image processing is a method to perform some operations on an image, for enhancing purposes or to extract some useful information from it [1]-[4]. In this paper a process of estimating the complexity of tri-dimensional (3D) surfaces of interest in different fields of research and the calculation of their fractal dimension from 2D images is presented. This represents a core-research area within engineering and computer science disciplines. The concept of "surface modeling" generally describes the process of representing a physical or artificial surface by a geometric model, namely a mathematical expression. For example, terrain models [5] give an overview over a landscape and are often fascinating and overwhelmingly beautiful works of artists who invest all their affection and an immense amount of work and knowhow, combined with a developed sense for the portrayed landscape. Many techniques can be applied for the characterization of a surface. Terrain modeling especially relates to the representation of the natural surface of the Earth. Cartographic terrain or relief models [6] are threedimensional representations of a part of the Earth's surface. They convey an immediate and direct impression of a landscape and are much easier to understand for most people than two-dimensional models. In contrast to virtual models 
generated by a computer [7], a physically present model is easier and faster to understand.

There are many reasons for the usefulness of the characterization of true surface areas, especially for surfaces with complex textures [8]. As an example, in the reconstruction of extensive landscape surfaces and largescale objects, the development of landscape units relies on the fusing of plot-scale derived data with landscape-scale measurements obtained from towers, aircraft, satellites, and Unmanned Aerial Vehicles (UAV) [9], [10].

The choice of a different approach, that is fractal geometry, for describing the surfaces is supported by the fact that many surfaces observed in nature are typically complex, irregular in shape and thus cannot be described completely by Euclidean geometry. The analysis of complex surfaces can be important and relevant also because in some applications can be useful to detect their changes due, for example, to wear, or to other several reasons depending on the typology of the surface.

Traditional fractal models usually assume the fractal nature of landforms to be homogeneous and describe their fractal properties as a uniform fractal dimension [11]. Fractal geometry [12] is becoming increasingly popular in different sciences to describe complex irregular objects. Models of fractal lines and surfaces were created to describe the complexity of different terrain surfaces [13]. Fractal dimension [14] is a property of fractals that is maintained over all magnifications and is therefore well-defined, but in addition, it also reveals the complexity of the fractal. In general, it is possible to calculate the fractal dimension [15] only on pure mathematical constructs.

Many natural phenomena exhibit some form of statistical self-similarity that can be modeled by fractal surfaces. Real surfaces also have varying statistical behavior from place to place, so, for example, sandy beaches do not exhibit the same fractal properties as mountain ranges. A fractal function, however, is statistically stationary, meaning that its bulk statistical properties are the same everywhere. Thus, any real approach to modeling real surfaces requires the ability to modulate fractal behavior spatially. Technically speaking, any surface in three-dimensional space has a topological dimension of 2 , and therefore any fractal surface in the three-dimensional space has a Harsdorf dimension of between 2 and 3. Fractals are shapes that look the same on all scales of magnification, that is they are self- affine. The statistical self-affinity of fractal shapes is inherent in the natural world and maybe exploited to simulate natural processes.

In this paper, a solution to the open problem of describing different complex surfaces is reported. The complex structure of each surface is described under the assumption of known coordinates of the surface. Also, a new method for estimating fractal dimension in $3 D$ space with the average space component of Hurst exponent $H$ and its application in the analysis of surfaces is presented. Different surfaces were considered and analyzed with the two proposed methods.

\section{MATERIALS AND METHODS}

In this section, the method implemented in this study is reported. Data collection and analysis methodologies are described. The characteristics and the relevance in different field of research of each surface considered for the application of the methods are also discussed.

\section{A. Data Collection and Proposed Method}

The surfaces chosen as the object of this research were selected because they are very complex to describe. Their parameters are inexpensive and available in a continuous fashion and can, therefore, be used to replace part of the expensive field sampling. Point Data for Digital Modeling consists of individual discrete $X, Y$ and $Z$ locations, without connecting features between them. For the analysis of the relative images of the surfaces, the software ImageJ [16] was used. Each image was imported into the software in JPEG format.

First, the $2 D$ images were converted into $3 D$ images.
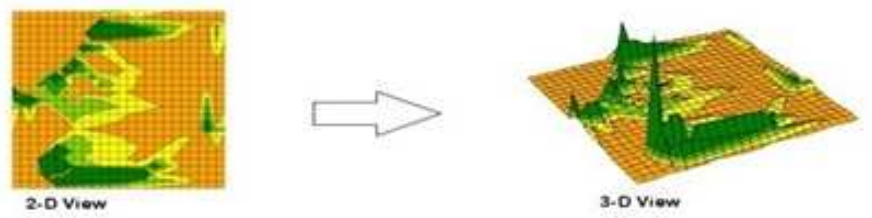

Fig. 1 Conversion of 2D image into 3D image.

Then, the coordinates $(x, y, z)$ of the $3 D$ images with the software ImageJ were found.

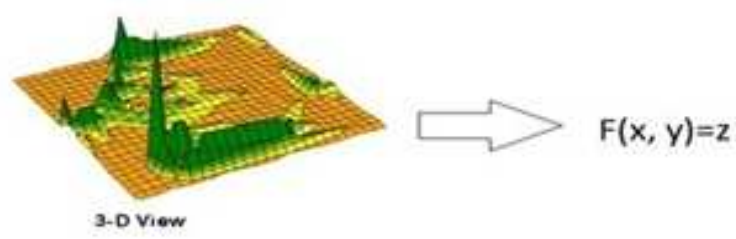

Fig. 2 Coordinates of a 3D image.

These coordinates were referred to a coordinate system $x y$. A random process is statistically evaluated using the Hurst parameter $H$ [17] or by determining the distribution function. The Hurst parameter $H$ as self-similarity criteria cannot be accurately calculated, but only estimated. In this study, a new method to estimate the Hurst exponent $H$ for a $3 D$ object was developed. There are several different methods [18] to produce estimates of the parameter $H$, which considered together more or less deviate. In doing so there are no criteria to determine which method can give the best result. Here, the $R / S$ method, adjusted rescaled range method [19] or adjusted scale, was used which is also a graphic method based on the properties of the Hurst phenomenon. One of new methods to estimate the Hurst exponent $H$ for $3 D$ objects was shown in [20]. There are two options. First, the Hurst exponent $H$ in $3 D$ space with average space component of graph $x z$ after $y$-axis is estimated. As mentioned before, the coordinates $(x, y, z)$ of the surfaces (images) were found. Then, only the $z$ coordinates to estimate the Hurst exponent $H$ were used. All $z$ coordinates present in the $2 D$ space component graph, are continuous. Also, all points $\left(x_{i}, y_{0}, z_{i}\right)$ present first space component series in $2 D$ graph for all points $\left(x_{i}, z_{i}\right)$. All points $\left(x_{i}, y_{1}, z_{i}\right)$ present second space component in $2 D$ graph for all points $\left(x_{i}, z_{i}\right)$. Space component for all $y_{i}, \forall i$ were made. Then, the Hurst exponent $H(x, y)$ for all these space components was estimated. After estimating the Hurst exponent $H(x, y)$, the 
average of the Hurst exponent $H(x, y)$ for all these space components was calculated by equation (1).

$$
H(x, y)=\left(H(x, y)_{1}+H(x, y)_{2}+\ldots+H(x, y)_{n}\right) / n
$$

The fractal dimension in $3 D$ space for the surface was calculated with the equation $D=3-H(x, y)$.

Subsequently, the Hurst exponent $H(y, x)$ in $3 D$ space with the average space component of graph $y z$ after $\mathrm{x}$-axis was estimated.

All coordinates $(x, y, z)$ of the surface were found. Then, only $z$ coordinates to estimate the Hurst exponent $H(y, x)$ were used. All $z$ coordinates presented in $2 D$ space component graph, are continuous. Also, all points $\left(x_{0}, y_{i}, z_{i}\right)$ present first space component series in $2 D$ graph for all points $\left(y_{i}, z_{i}\right)$. All points $\left(x_{1}, y_{i}, z_{i}\right)$ present second space component in $2 D$ graph for all points $\left(y_{i}, z_{i}\right)$. Space component for all $x_{i}, \forall i$ were made. Then, the Hurst exponent $H(y, x)$ for all these space components was estimed. After estimating the Hurst exponent $H(y, x)$, the average of the Hurst exponent $H(y, x)$ for all these space components (Fig. 2) was calculated by equation (2).

$$
H(y, x)=\left(H(y, x)_{1}+H(y, x)_{2}+\ldots+H(y, x)_{n}\right) / n
$$

The case study approach is a highly appropriate method for the analysis of surfaces. The basic Hurst exponent can be related to the expected size of changes, as a function of the lag between observations. For both methods of estimating Hurst exponent $H$, it is possible to calculate the fractal dimension in $3 D$ space for a surface with the equation $D=3$ $H$.

\section{B. Analyzed Surfaces}

1) Surface of desert: In recent years surface fractal exploration has demonstrated special advantages and successful applications to study surface structure for desert areas. A desert pavement is a desert surface that is covered with closely packed, interlocking angular or rounded rock fragments of pebble and cobble size [21]. A desert is a type of landscape that can be made up of nothing but sand. Formally identified, this is a certain type of terrain that is known for its dryness due to the lack of rain. Everything from the sand patterns to the general calmness of the sandy terrain can easily soothe the eyes. The percolation of infrequent precipitation tends to cause lateral and down slope movement of silt particles beneath the surface of the ground. This leads to the concentration of gravel, a process enhanced by the constant removal of fine sediment at the surface by wind action. Thus, pavements are produced by the combined effects of water and wind.

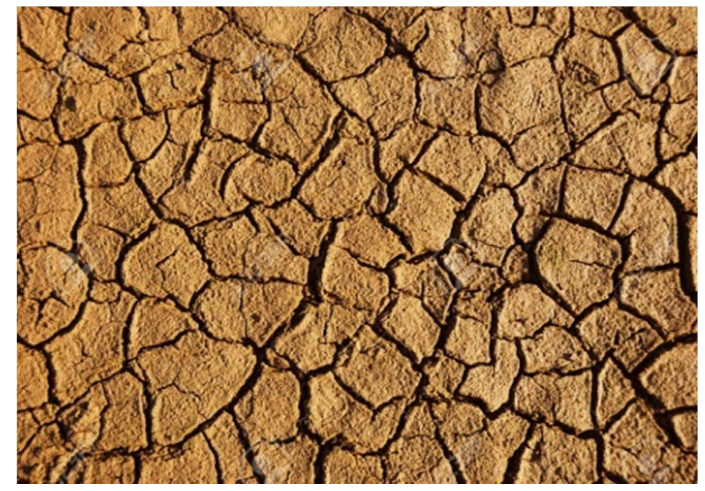

Fig.3 Surface of desert.

Evaporation and capillarity draw soil moisture to the surface and may precipitate calcium carbonate, gypsum, and other salts that cement the pebbles together to form a desert conglomerate. Thus, exogenous forces of nature impact on design of geometry surfaces. The surface of desert analyzed in this study is reported in Fig.3.

2) Surface of hardened material: Surface microstructure and composition of a material play a crucial role in determining the surface dependent engineering properties (wear, corrosion and oxidation resistance) of a mechanical component. In an era of increased emphasis on sustainability and quality assurance, knowledge about metals and other materials used in products, manufacturing processes and construction is invaluable. Metallurgy is a domain of materials science and engineering that studies the physical and chemical behavior of metallic elements [22]. Surface engineering aims at the orientation of the microstructure and composition of the near surface region of a component without affecting the bulk material. Objects observed in the microstructure of heat treatment materials are typically complex, irregular in shape and thus, cannot be described completely by Euclidean geometry. Fractal geometry can be used in material science to describe these complex irregular objects.

For the analysis with the proposed methodologies of this study, the surface of material hardened with robot laser cell with $6 \mathrm{~mm} / \mathrm{s}$ at $1050^{\circ} \mathrm{C}$ was considered. The analyzed image is reported in Fig. 4.

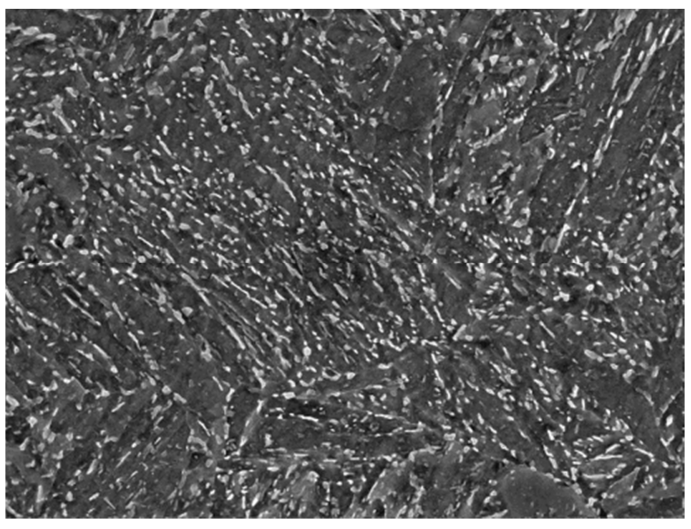

Fig.4 Surface of a hardened material. 
3) Surface of bone: Trabecular bone microstructure is an important factor influencing bone strength, which in turn is the most important bone parameter indicating bone fracture risk. Microarchitectural bone imaging is a nondestructive, noninvasive, and precise methodology with which both the apparent density and trabecular microstructure of intact bones and bone samples can be assessed in a single measurement. The procedure can help improve predictions of fracture risk, clarify the pathophysiology of skeletal diseases, and define the response to therapy and interventions. In this field, the new proposed method for estimating fractal dimension in $3 D$ space can be useful to describe the complex surface of the bone. Fig. 5 shows the surface of bone analyzed in this study.

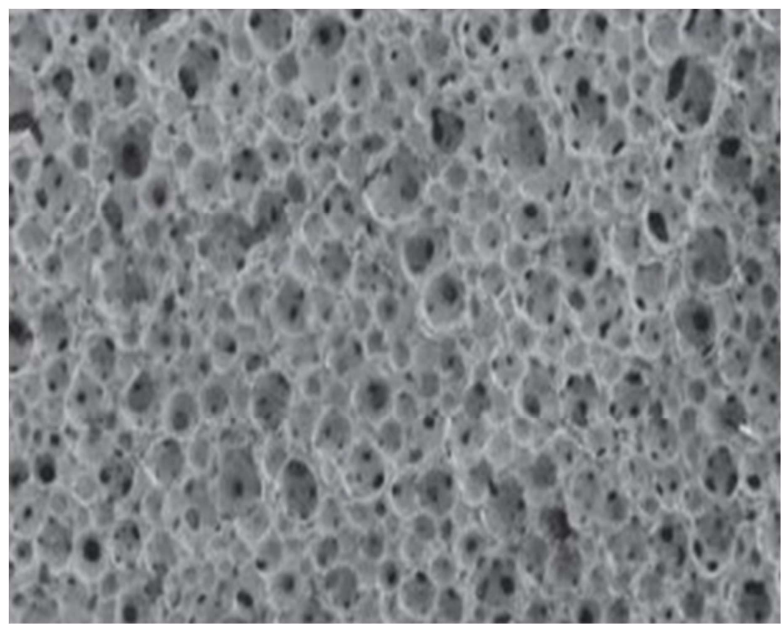

Fig.5 Surface of bone.

4) Surface of alluvial fan: Alluvial (torrential) fans are fan-shaped debris strewn by mountain streams at the passage from steep grape into the bottom of the valley. It is a sediment of a mountain stream that, due to the reduced steepness and speed at the transition to a more laid-back world, also reduces the power of carrying the material. Water in the stream does not erode, but accumulates material. Especially in mountain resorts, fattening is of great importance for settlement, thus the study of alluvial fans has a practical relevance. The image of the surface of an alluvial fan analyzed in this study is reported in Fig.6.

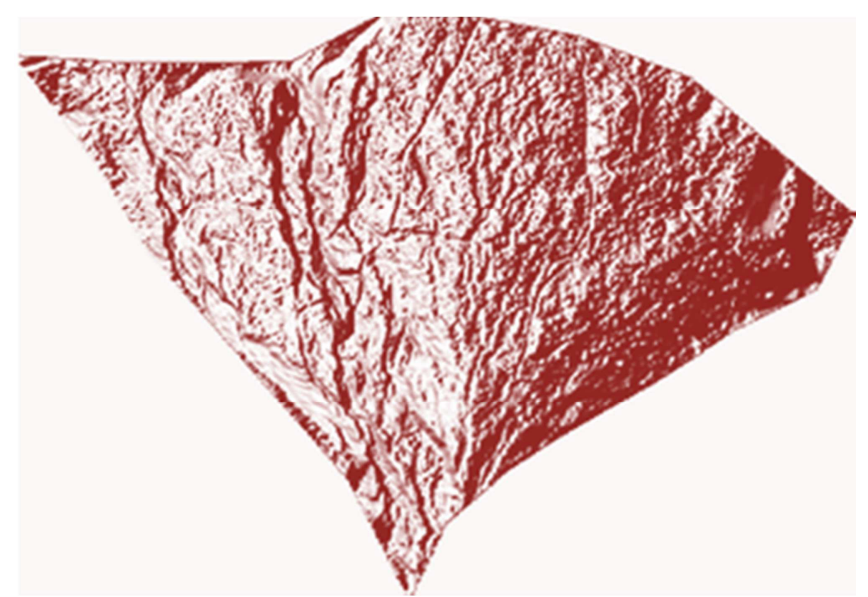

Fig. 6 Surface of alluvial fan.

\section{RESULTS AND DISCUSSION}

The proposed new methods were applied to the different surfaces also comparing them by means of the calculated parameters. With these methods of fractal geometry, irregular and complex structure of four different surfaces, namely, the surface of desert, the surface of hardened material, the surface of bone and, the surface of an alluvial fan was described.

First, the Hurst exponent $H$ in $3 D$ space with an average space component of graph $x z$ after $y$-axis was estimated. Secondly, the procedure was repeated with an average space component of graph $y z$ after $x$-axis.

Table I reports Hurst exponent $H$ and fractal dimension for the different surfaces considered. The first column reports the results obtained for the surface of desert, the second column the results for the surface of hardened material, the third column portrays the results for the surface of bone and, finally, the fourth column presents the results for the surface of an alluvial fan.

TABLE I

HURST EXPONENT H AND FRACTAL DIMENSION FOR THE DIFFERENT SURFACES

\begin{tabular}{|l|c|c|c|c|}
\hline & $\begin{array}{c}\text { Surface } \\
\text { of desert }\end{array}$ & $\begin{array}{c}\text { Surface of } \\
\text { hardened } \\
\text { material }\end{array}$ & $\begin{array}{c}\text { Surface } \\
\text { of bone }\end{array}$ & $\begin{array}{c}\text { Surface of } \\
\text { alluvial fan }\end{array}$ \\
\hline $\mathrm{H}_{\mathrm{XY}}$ & 0.51 & 0.09 & 0.34 & 0.42 \\
\hline $\mathrm{H}_{\mathrm{YX}}$ & 0.56 & 0.16 & 0.36 & 0.49 \\
\hline $\mathrm{FD}_{\mathrm{XY}}$ & 2.49 & 2.91 & 2.66 & 2.58 \\
\hline $\mathrm{FD}_{\mathrm{YX}}$ & 2.44 & 2.84 & 2.64 & 2.51 \\
\hline
\end{tabular}

In Table I, $H_{X Y}$ represents the average of the Hurst exponent $H$ for all space components by the $y$-axis. $F D_{X Y}$ represents the fractal dimension calculated by $F D=3-H_{X Y}$ and, $H_{Y X}$ represents the average of the Hurst exponent $H$ for all space components by the $x$-axis. $F D_{Y X}$ represents the fractal dimension calculated by $F D=3-H_{Y X}$.

From this table it is possible to observe that each analyzed surface has a different Hurst exponent $H$ and, consequently, a different fractal dimension. This means that the complexity of the different surfaces can be characterized by the considered parameters.

The results of the table highlight that Hurst exponents $H$ ${ }_{X Y}$ are smaller with respect to the Hurst exponents $H_{Y X}$. Thus, fractal dimensions $F D_{X Y}$ are higher, like the fractal dimensions $F D_{Y X}$. Smaller holes in the structure bring smaller Hurst exponent $H$ and consequently higher dimension.

Most of surfaces of interest for research are very complex and difficult to measure. Fractals are now used in many forms to create textured landscapes and other intricate models. It is possible to create all sorts of realistic fractal forgeries, images of natural scenes, such as mountain ranges and coastlines, to name but a few. The new method developed for estimating the fractal dimension in $3 D$ space with an average space component can be a useful tool in different field of research.

The fractal approach is appropriate in the characterization of complex and irregular surfaces of different origin and can be effectively utilized for predicting the properties of surfaces from fractal dimensions. The fractal analysis of different digitized surfaces indicates that useful correlations 
can be derived between the fractal dimensions and the surface features. The degree of complexity depends on the surface, being higher in the surface of hardened material and minimum in the surface of desert.

Methods for estimating Hurst exponent $H$ and their application in $2 D$ space are known, but in this paper, the approach is new. According to the data of a major database, over 1500 scientific publications on fractal relative to surfaces have been published on journals until now. Theoretical implications of results and practical application are, however, presented in this paper.

As an example, the surface of desert analyzed in this paper can be used to model how exogenous and endogenous forces impact into relief of terrain surfaces on Earth. Another application of the proposed method is relative to the surface of hardened material. In the field of materials research the method can be useful for the modelling of topographical properties of materials after heat treatment process. The application relative to the field of medicine can be useful in orthopedics, to evaluate the complexity of a fracture of bone, or similar injuries. Finally, in the study of alluvial fan, surface modelling with fractals can help to solve security issue of people's urban environment.

\section{CONCLUSIONS}

Analyzing the complexity of surfaces is a very hard problem, which is of interest in different fields of research, e.g., biomimetics [23], virtual reality [24], medical applications [25], [26], energy production [27], animal monitoring [28], [29]. The paper reports two new methods for estimating the fractal dimension in 3D space with an average space component, and its application in analyzing complex surfaces. Various surfaces with both methods were analyzed and the results were compared.

The two methods give different results and confirm the initial hypothesis stated by the authors that with known coordinates of a surface, it is possible to describe its complex structure.

The main findings can be summarized by the following points:

- New methods for estimating the Hurst exponent $H_{X Y}$ in $3 D$ space with an average space component of graph $x z$ after $y$-axis and for estimating the Hurst exponent $H_{Y X}$ in $3 D$ space with an average space component of graph $y z$ after $x$-axis were developed. Also, a new concept for estimating complexity of a 3D structure with known coordinates was presented.

- The two new methods for estimating the Hurst exponent $H$ in $3 D$ space were compared. Estimation of the Hurst exponent $H_{X Y}$ gives higher values with respect to the estimates of the-Hurst exponent $H_{Y X}$. This means that the method of estimation of Hurst exponent $H_{Y X}$ considers higher complexity of surfaces, because this process gives higher fractal dimension of surfaces.

- The new method was applied to describe the complexity of different surfaces. There are many other different complex surfaces of interest for research in different fields, and classical tool are not able to describe them. The proposed method can be potentially useful to overcome the limitations of other procedures, and can have applications to several 3D structures and different purposes such as pattern recognition, computer graphics, and computational geometry.

In conclusion, surface complexity analysis is proving to be central for many applications and different approaches are being applied to the related computer-aid design tools, as Bspline [30], Bezier mathematical techniques [31] and machine learning [32], [33]. The latter, representing a current and growing field of research in a multitude of areas ([34]-[37]) would be certainly promising in the area of surface complexity analysis [38]. In this framework, deep learning techniques, i.e., convolutional neural networks [39]-[41] which solve the central problem of representation learning by building complex features out of simpler features, could benefit of the proposed approach.

\section{ACKNOWLEDGMENT}

This research was supported by a funding from the University of Catania, research programme 2019/2021 and partially financed by the Slovenian research agency through research project $\mathrm{J} 7-8273$.

\section{REFERENCES}

[1] J. C. Russ, F. B. Neal, The image processing handbook, 17th ed., CRC Press, Francis and Taylor group, Boca Raton, U.S., 2017.

[2] M. Calì, S. M. Oliveri, A. Gloria, M. Martorelli, and D. Speranza. "Comparison of commonly used sail cloths through photogrammetric acquisitions, experimental tests and numerical aerodynamic simulations." Procedia Manufacturing, 11, pp. 1651-1658, 2017.

[3] M. Calì, S. M. Oliveri, U. Cella, M. Martorelli, A. Gloria, \& D Speranza. "Mechanical characterization and modeling of downwind sailcloth in fluid-structure interaction analysis." Ocean Engineering, 165, pp. 488-504, 2018.

[4] F. Beritelli, et al. "Automatic heart activity diagnosis based on Gram polynomials and probabilistic neural networks." Biomedical engineering letters 8.1: pp. 77-85, 2018.

[5] C. Wei, C. Zhanchuan, T. Zesheng. "Fractal structure of lunar topography: An interpretation of topographic characteristics." Geomorphology, Volume 238, 1 pp. 112-118, June 2015.

[6] M. K. Annette. "Critical cartography 2.0: From participatory mapping to authored visualizations of power and people." Landscape and Urban Planning 142 pp. 215-225, 2015.

[7] L. Zhu, Z. He, X. Pann, X. Wu. "An Approach to Computer Modeling of Geological Faults in 3D and an Application." Journal of China University of Mining and Technology. Volume 16, Issue 4, pp. 461-465, December 2006.

[8] A. B. Murray, E. Lazarus, A. Ashton, A. Baas, G. Coco, T. Coulthard, \& J. Pelletier. "Geomorphology, complexity, and the emerging science of the Earth's surface." Geomorphology, 103(3), pp. 496-505, 2009.

[9] M. Calì, R. Ambu, "Advanced 3D Photogrammetric Surface Reconstruction of Extensive Objects by UAV Camera Image Acquisition", Sensors 18(9), 2815, Aug.2018.

[10] L. A. Méndez-Barroso, J.L. Zárate-Valdez, A. Robles-Morúa, "Estimation of hydromorphological attributes of a small forested catchment by applying the Structure from Motion (SfM) approach", International Journal of Applied Earth Observation and Geoinformation, vol.69, pp. 186-197, July 2018.

[11] Xu T., I.D. Moore, J.C. Gallant. "Fractal dimensions and landscapes - a review. Geomorphology, 8, pp. 245-262, 1993.

[12] X. H.Shen, L. J.Zou, G. F. Zhang, N. Su, W.Y. Wu, S. F. Yang. "Fractal characteristics of the main channel of Yellow River and its relation to regional tectonic evolution." Geomorphology, Volume 127, Issues 1-2, pp. 64-70, April 2011.

[13] A. C. W. Baas "Chaos, fractals and self-organization in coastal geomorphology: simulating dune landscapes in vegetated environments." Geomorphology, Volume 48, Issues 1-3, pp. 309-328, November 2002. 
[14] B. B. Mandelbrot. "The fractal geometry of nature." New York: W. H. Freeman, 93, 1998.

[15] C. Zhiying, L. Yong and Z. Ping, "A comparative study of fractal dimension calculation methods for rough surface profiles." Chaos, Solitons \& Fractals, vol.112, pp.24-30, July 2018.

[16] F. Tiago, R. Wayne. ImageJ. User GuidIJ1. 46r. 2002 http://imagej.nih.gov/ij/docs/guide/user-guide.pdf.

[17] S. Ouchi, M. Matsushita. "Measurement of self-affinity on surfaces as a trial application of fractal geometry to landform analysis." Geomorphology, Volume 5, Issues 1-2, pp. 115-130, May 1992.

[18] J. Qin, Z. Deyu, W. Guangqian, L. N. Sai. "Influence of particle shape on surface roughness: Dissimilar morphological structures formed by man-made and natural gravels." Geomorphology, Volume 190, pp. 16-26, May 2013.

[19] Q.C. Sung, Y.C. Chen. "Self-affinity dimensions of topography and its implications in morphotectonics: an example from Taiwan." Geomorphology, Volume 62, Issues 3-4, 1, pp. 181-198, Oct. 2004.

[20] M. Babič, P. Kokol, N. Guid, P. Panjan. "A new method for estimating the Hurst exponent $\mathrm{H}$ for $3 \mathrm{D}$ objects." Materiali in tehnologije 48-2, 2014.

[21] Phillips, J.D.Earth Surface Systems: Complexity, Order and Scale. Blackwell, Oxford, 1999.

[22] Capizzi, Giacomo, et al. "A multithread nested neural network architecture to model surface plasmon polaritons propagation." Micromachines 7.7 (2016): 110.

[23] C. Kumar, A. Palacios, V. A. Surapaneni, G. Bold, M. Thielen, E. Licht, T. E. Higham, T. Speck and V. Le Houérou. "Replicating the complexity of natural surfaces: technique validation and applications for biomimetics, ecology and evolution, 377." Philosophical Transactions of the Royal Society A: Mathematical, Physical and Engineering Sciences

[24] H. Sun \& H. Chen. "Soft-Touch Haptics Modeling of Dynamic Surfaces." Virtual Technologies for Business and Industrial Applications: Innovative and Synergistic Approaches. Editors: N. Raghavendra Rao (VIT University, India), 2011.

[25] C. H. Yan, S. H. Ong, Y. Ge, J. Zhang, S. H. Teoh and B. H. Okker, "A neural network approach for 3D surface modeling and registration," IEEE International Workshop on Biomedical Circuits and Systems, Singapore, pp. S3/2-17, 2004.

[26] I. P. Nanda, M. Hazwan Hassim, M. Hasbullah Idris, M. H. Jahare, A. Arafat. "Effect of Mechanical Tumbling Parameters on Surface Roughness and Edge Radius of Medical Grade Cobalt Chromium Alloy." International Journal on Advanced Science, Engineering and Information Technology, Vol. 9 No. 1, 2019.

[27] S. Brusca, G. Capizzi, G. Lo Sciuto, and G. Susi. "A new design methodology to predict wind farm energy production by means of a spiking neural network based-system." International Journal of Numerical Modelling: Electronic Networks, Devices and Fields, 7 2017.
[28] P. Loreti, A. Catini, M. De Luca, L. Bracciale, G. Gentile and C. Di Natale. "The Design of an Energy Harvesting Wireless Sensor Node for Tracking Pink Iguanas". Sensors, 19(5), 985, 2019.

[29] L. Bracciale, A. Catini, G. Gentile, P. Loreti. "Delay tolerant wireless sensor network for animal monitoring: The Pink Iguana case" Lecture Notes in Electrical Engineering, 429, pp. 18-26, 2017.

[30] R. Zakaria, A. Wahab, \& R. U. Gobithaasan. "Fuzzy B-Spline surface modeling." Journal of Applied Mathematics, 2014.

[31] A. Gálvez and A. Iglesias. "Firefly Algorithm for Polynomial Bézier Surface Parameterization." Journal of applied mathematics, 2013.

[32] U. Reuter, A. Sultan and D. Reischl. "A comparative study of machine learning approaches for modeling concrete failure surfaces." Advances in Engineering Software Volume 116, pp. 67-79, February 2018.

[33] C. T. Yeu, M. Lim and G. Huang, "Terrain Modeling Using Machine Learning Methods," 9th International Conference on Control, Automation, Robotics and Vision, pp. 1-4, Singapore, 2006.

[34] S. Angra and S. Ahuja, "Machine learning and its applications: A review,"IEEE International Conference on Big Data Analytics and Computational Intelligence (ICBDAC), pp. 57-60, Chirala, 2017.

[35] Cardarilli G.C. et al. "Efficient Ensemble Machine Learning Implementation on FPGA Using Partial Reconfiguration," Applications in Electronics Pervading Industry, Environment and Society. ApplePies 2018. Lecture Notes in Electrical Engineering, vol 550. Springer, Cham.

[36] G. Susi, L. Anton Toro, L. Canuet, M. E. Lopez, F. Maestu, C. R. Mirasso, and E. Pereda, "A neuro-inspired system for online learning and recognition of parallel spike trains, based on spike latency, and heterosynaptic STDP”. in Frontiers in Neuroscience, 12:780, 2018. ISSN 1662-453X. doi: 10.3389/fnins.2018.00780.

[37] M. Elangovan, N. R. Sakthivel, S. Saravanamurugan, Binoy. B. Nair, V. Sugumaran. "Machine Learning Approach to the Prediction of Surface Roughness Using Statistical Features of Vibration Signal Acquired in Turning." Procedia computer science, 2015.

[38] D. Racki, D. Tomazevic, D. Skocaj. "A Compact Convolutional Neural Network for Textured Surface Anomaly Detection" IEEE Winter Conference on Applications of Computer Vision (WACV). 2018.

[39] M. Matta, G. C. Cardarilli, L. Di Nunzio, R. Fazzolari, D. Giardino, M. Re, F. Silvestri, S. Spanò, "Q-RTS: a real-time swarm intelligence based on multi-agent Q-learning." Electronics Letters, 55 (10), pp. 589-591, 2019.

[40] G. C. Cardarilli, L. Di Nunzio, R. Fazzolari, D. Giardino, M. Matta, M. Re, F. Silvestri and S. Spanò "Efficient Ensemble Machine Learning implementation on FPGA using Partial Reconfiguration." Lecture Notes in Electrical Engineering. 2019 ARTICLE IN PRESS.

[41] G. C. Cardarilli, L. Di Nunzio, R. Fazzolari, M. Re, S. Spanó "AWSOM, an Algorithm for High-speed Learning in Hardware SelfOrganizing Maps" IEEE Transactions on Circuits and Systems II: Express Briefs DOI:10.1109/TCSII.2019.2909117, 2019 ARTICLE IN PRESS. 